\title{
Morphological stability diagram for slowly and rapidly solidifying binary systems
}

\author{
Peter K. Galenko ${ }^{1,2, a}$ and Denis A. Danilov ${ }^{3}$ \\ ${ }^{1}$ Friedrich-Schiller-Universität-Jena, Faculty of Physics and Astronomy, Otto Schott \\ Institute of Materials Research, 07743 Jena, Germany \\ ${ }^{2}$ Ural Federal University, Department of Theoretical and Mathematical Physics, \\ Laboratory of Multi-Scale Mathematical Modeling, 620000 Ekaterinburg, Russia \\ ${ }^{3}$ Udmurt State University, Physical Faculty, 426034 Izhevsk, Russia
}

Received 13 June 2019 / Received in final form 28 June 2019

Published online 11 February 2020

\begin{abstract}
A linear morphological stability of the solid-liquid interface is analyzed for a binary alloy in the limit of low and high crystal growth velocities. Using the result of this analysis, a diagram of morphologies is derived for a whole range of solidification rates with indicating critical growth velocities for the transitions planar front $\Leftrightarrow$ cellular/dendritic structure. It is specially noted that the speed of solute diffusion in the bulk liquid limits the absolute chemical stability velocity from the high-rate transition cells/dendrites $\Rightarrow$ planar front.
\end{abstract}

\section{Introduction}

Originating of two-phase mushy zone, as a heterogeneous zone of phase transition between liquid and solid [1,2], occurs as a response of crystallizing liquer/solution/ melt on the existing undercooling for the fastest decrease of this undercooling (see Fig. 1). Indeed, an intensive atoms attachment to the solid-liquid interface leads to the fast decrease in the interface undercooling with the formation of crystalline ensemble of cellular or dendritic morphology (see Refs. [4-6] and references therein). In appearance of such morphologies a key role plays an establishing balance between destabilizing and stabilizing forces which are competing at the interface leading it to the planar or cellular/dendritic form. Therefore, the originating and development of two-phase mushy zone are going through the stages of front perturbation on the microscopic spatial level and formation of mesoscopic structures of cellular-dendritic morphology.

In the present work, we consider linear morphological stability of solidification front for definition of its morphology for a given growth velocity. This problem takes its origination from the classical works of Mullins and Secerka $[7,8]$ as well as from the works of Coriell et al. [9-12]. Later on, this morphological analysis has been extended to the non-linear range by Davis [13] and generalized to the region of large growth velocity by Trivedi and Kurz [14-16]. Extended analysis of morphological stability to the case of local non-equilibrium solidification [17] was necessary to explain experimental results on crystallization of liquids undercooled up to $400-500 \mathrm{~K}$ and solidifying with the velocities $1-100 \mathrm{~m} / \mathrm{s}$ [18]. Therefore, we present analysis which

${ }^{a}$ e-mail: peter.galenko@uni-jena.de 


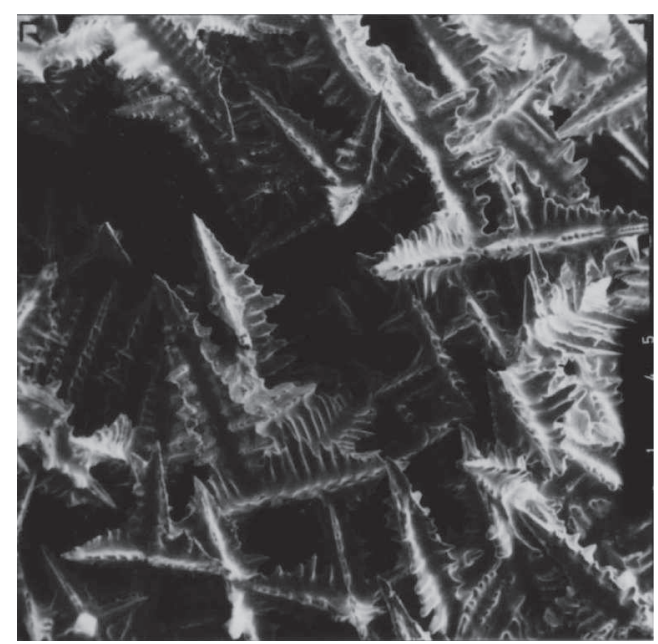

Fig. 1. Dendritic structure within the two-phase mushy zone. This structure has been obtained by decantation of a residual interdendritic melt upon primary solidification of Pb-Sn alloy's droplets [3].

allows us to construct the diagram of morphological stability from very small velocity of crystals growing at the near equilibrium conditions up to the high velocity at which diffusionless (chemically partitionless) solidification may occur.

\section{Criterion of the morphological stability}

When a harmonic perturbation with the wavelength $\lambda$ and the corresponding frequency

$$
\omega=2 \pi / \lambda
$$

is applied to a moving planar interface, the resulting criterion of its marginal stability is given by [17]

$$
\begin{aligned}
& \Gamma \omega^{2}+K_{L} G_{L} \xi_{L}+K_{S} G_{S} \xi_{S}-m_{v} G_{C} \xi_{C}=0, \quad V<V_{D}, \\
& \Gamma \omega^{2}+K_{L} G_{L} \xi_{L}+K_{S} G_{S} \xi_{S}=0, \quad V \geq V_{D},
\end{aligned}
$$

where indices " $L$ " and " $S$ " are related to the liquid or solid, respectively, $G$ is the thermal gradient, $K$ is the thermal conductivity, $V$ the velocity of the interface, $V_{D}$ is the solute diffusion speed in bulk liquid, $\Gamma$ is the Gibbs-Thomson coefficient related to the capillary of the perturbed interface, and $m_{v}$ is the velocity dependent slope of the liquidus line (so-called "nonequilibrium liquidus in the kinetic diagram of a phase state"). The stability functions $\xi$ in equation (2) are described by

$$
\begin{aligned}
\xi_{L} & =\frac{\omega_{L}-V / a_{L}}{K_{L} \omega_{L}+K_{S} \omega_{S}}, \\
\xi_{S} & =\frac{\omega_{S}+V / a_{S}}{K_{L} \omega_{L}+K_{S} \omega_{S}}, \\
\xi_{C} & =\frac{\omega_{C}-V /\left[D\left(1-V^{2} / V_{D}^{2}\right)^{1 / 2}\right]}{\omega_{C}-\left(1-k_{v}\right) V /\left[D\left(1-V^{2} / V_{D}^{2}\right)^{1 / 2}\right]}, \quad V<V_{D},
\end{aligned}
$$




$$
\xi_{C}=0, \quad V \geq V_{D},
$$

where $a$ is the thermal diffusivity, $D$ the diffusion constant and $k_{v}$ is the velocity dependent solute partitioning function related to nonequilibrium redistribution of atoms on the interface. Frequencies $\omega$ have the following form

$$
\begin{aligned}
& \omega_{L}=\frac{V}{2 a_{L}}+\left[\left(\frac{V}{2 a_{L}}\right)^{2}+\omega^{2}\right]^{1 / 2}, \\
& \omega_{S}=-\frac{V}{2 a_{S}}+\left[\left(\frac{V}{2 a_{S}}\right)^{2}+\omega^{2}\right]^{1 / 2}, \\
& \omega_{C}=\frac{V}{2 D\left(1-V^{2} / V_{D}^{2}\right)^{1 / 2}}+\left[\left(\frac{V}{2 D\left(1-V^{2} / V_{D}^{2}\right)^{1 / 2}}\right)^{2}+\omega^{2}\right]^{1 / 2} .
\end{aligned}
$$

In the local equilibrium limit, $V_{D} \rightarrow \infty$, criterion (2) together with equations (3)-(8) transforms into the criterion of marginal stability of Trivedi and Kurz [14]. The introduction of the finite diffusion speed, $V_{D}$, into the model leads to the principal result, which is related to the transition to diffusionless (chemically partitionless) solidification. It can be shown using analytical solution for the atomic redistribution around the interface. Indeed, one can obtain two solutions from the only diffusion equation. These describe two different regimes: regime with atomic diffusion at $V<V_{D}$ and regime with the absence of atomic diffusion at $V \geq V_{D}$. Particularly, around the perturbed interface, the field of solute concentration is given by [17]

$$
\begin{aligned}
C-C_{\infty}= & \frac{G_{C} D\left(1-V^{2} / V_{D}^{2}\right)}{V}\left[1-\exp \left(-\frac{V z}{D\left(1-V^{2} / V_{D}^{2}\right)}\right)\right] \\
& +\left(b-G_{C}\right) \delta(t) \sin (\omega x) \exp \left(-\frac{\omega_{C} z}{\left(1-V^{2} / V_{D}^{2}\right)^{1 / 2}}\right), \quad V<V_{D}, \\
C-C_{\infty}= & 0, \quad V \geq V_{D},
\end{aligned}
$$

where $C_{\infty}$ is the nominal (initial) concentration of a binary system which is considered as far-field concentration. Solution (9) shows that with the finite interface velocity $V \geq V_{D}$, the solute diffusion ahead of the rapid interface is absent. From this it follows that the destabilizing contribution due to concentration gradient $G_{C}$ in equation (2) is absent at $V \geq V_{D}$. In such a case, the balance for morphological stability is defined by the relation of the stabilizing force $\Gamma \omega^{2}$, due to surface energy, and the contribution $K_{L} G_{L} \xi_{L}+K_{S} G_{S} \xi_{S}$ of temperature gradients, $G_{L}>0$ and $G_{S}>0$, which are positive in the case of directional solidification.

\section{Analysis of the stability function}

We are interested in the analysis of the concurrence of contribution of the surface energy and destabilizing concentration gradient which is true for $V<V_{D}$. Therefore, consider the first equation from the criterion (2). For this equation, one can introduce the following stability function (see, for details, Ref. [12]):

$$
S\left(\omega^{2}\right)=-\Gamma \omega^{2}-K_{L} G_{L} \xi_{L}-K_{S} G_{S} \xi_{S}+m G_{C} \xi_{C} .
$$

Taking the stability functions from equations (3) and (4) we assume equal coefficients of thermal diffusivity and of thermal conductivity in both phases, i.e., $a_{L}=a_{S}=a$ 
and $K_{L}=K_{S}=K_{T}$. Then we get

$$
K_{L} G_{L} \xi_{L}+K_{S} G_{S} \xi_{S}=\frac{G_{L}+G_{S}}{2}+\frac{G_{S}-G_{L}}{2} \frac{V}{2 a} \frac{1}{\sqrt{(V / 2 a)^{2}+\omega^{2}}} .
$$

As a result, the function (10) can be re-written as

$$
S\left(\omega^{2}\right)=-\Gamma \omega^{2}-\frac{G_{L}+G_{S}}{2}-\frac{G_{S}-G_{L}}{2} \frac{V}{2 a} \frac{1}{\sqrt{(V / 2 a)^{2}+\omega^{2}}}+m G_{C} \xi_{C}\left(\omega^{2}\right) .
$$

This function has two limiting cases: $\omega^{2}=0$ and $\omega^{2} \rightarrow \infty$. First, at $\omega^{2}=0$ one has $\xi_{C}=0$, and for the positive temperature gradients, $G_{L}>0$ and $G_{S}>0$, we have from equation (12) the following function

$$
S\left(\omega^{2}\right)=-\frac{G_{L}+G_{S}}{2}-\frac{G_{S}-G_{L}}{2}<0 .
$$

Second, at $\omega^{2} \rightarrow \infty$, one gets from equation (12) that $S\left(\omega^{2}\right) \rightarrow-\infty$.

Now let us analyze extremal points of the function $S\left(\omega^{2}\right)$ given by equation (12). With this aim we write the derivative $\partial S\left(\omega^{2}\right) / \partial \omega^{2}$ as follows

$$
\begin{aligned}
\frac{\partial S\left(\omega^{2}\right)}{\partial \omega^{2}}= & -\Gamma+\frac{G_{S}-G_{L}}{2} \frac{V}{2 a} \frac{1 / 2}{\left((V / 2 a)^{2}+\omega^{2}\right)^{3 / 2}} \\
& +\frac{m G_{C}}{2} \frac{1}{\left(\left(V /\left(2 D \sqrt{1-V^{2} / V_{D}^{2}}\right)\right)^{2}+\omega^{2}\right)^{1 / 2}} \\
& \times \frac{k V /\left(D \sqrt{1-V^{2} / V_{D}^{2}}\right)}{\left(\omega_{C}-(1-k) V /\left(D \sqrt{1-V^{2} / V_{D}^{2}}\right)\right)^{2}} .
\end{aligned}
$$

From this derivative one can find both asymptotic regimes for rapid solidification and slow solidification.

\subsection{The first asymptotic: a case of rapid solidification}

It can be seen from equation (14), that the derivative $\partial S\left(\omega^{2}\right) / \partial \omega^{2}$ is a monotonically diminishing function of $\omega^{2}$. Accordingly, if $\partial S\left(\omega^{2}\right) / \partial \omega^{2}<0$ at $\omega^{2}=0$, then we get $\partial S\left(\omega^{2}\right) / \partial \omega^{2}<0$ for any $\omega^{2}$. In such a case, the function $S\left(\omega^{2}\right)$ has not extremal points and it is the negative function, $S\left(\omega^{2}\right)<0$, for any $\omega^{2}$ in a positive temperature gradient [see Eq. (13)]. Thus, for this case, the planar interface is morphologically stable.

For the zero frequency, i.e., $\omega^{2}=0$, we get the following condition

$$
\frac{\partial S\left(\omega^{2}\right)}{\partial \omega^{2}}=-\Gamma+\frac{G_{S}-G_{L}}{4}\left(\frac{2 a}{V}\right)^{2}+\frac{m_{v} G_{C} D^{2}\left(1-V^{2} / V_{D}^{2}\right)}{k_{v} V^{2}}<0 .
$$

Omitting the terms with temperature gradients, one can get the stability condition in the following form

$$
\Gamma>\frac{m_{v} G_{C} D^{2}\left(1-V^{2} / V_{D}^{2}\right)}{k_{v} V^{2}} .
$$


From this it follows that the solidification planar front is stable if the contribution from the interfacial energy (proportional to $\Gamma$ ) is greater than the destabilizing contribution (proportional to the concentration gradient $G_{C}$ ).

Now, taking into account expression for the concentration gradient $G_{C}$ at the unperturbed interface [19]

$$
\begin{aligned}
& G_{C}=-\frac{\left(1-k_{v}\right) V C_{\infty}}{k_{v} D\left(1-V^{2} / V_{D}^{2}\right)}, \quad V<V_{D}, \\
& G_{C}=0, \quad V \geq V_{D}
\end{aligned}
$$

and using equation (16), one can obtain the following inequality

$$
\frac{k_{v}^{2} \Gamma}{m_{v}\left(k_{v}-1\right) C_{\infty}}>\frac{D}{V}
$$

This stability condition has the following meaning [20]. As soon as the characteristic diffusion length $D / V$ becomes smaller than the characteristic scale $k_{v}^{2} \Gamma /\left(m_{v}\left(k_{v}-1\right) C_{\infty}\right)$ of interfacial capillary, the planar interface becomes morphologically stable in rapid solidification regime.

The balance between the stabilizing and destabilizing actions [see Eq. (16)] or equality of the two lengths [see Eq. (18)] can be re-written in the following convenient form

$$
V_{A}=\frac{m_{v} D\left(k_{v}-1\right) C_{\infty}}{\Gamma k_{v}^{2}} \equiv \frac{D \Delta T_{v}}{k_{v} \Gamma}<V_{D}
$$

where

$$
\Delta T_{v}=m_{v} \frac{k_{v}-1}{k_{v}} C_{\infty}
$$

is the velocity dependent interval of solidification (in the kinetic phase diagram). This is the velocity $V_{A}$ for absolute chemical stability above which the planar interface becomes stable. The form of equation (19) coincides with the expression given for the case of local equilibrium solute diffusion transport at $V_{D} \rightarrow \infty$ [14]. However, a final form of the function $V_{A}\left(C_{\infty}\right)$ is defined by the functions of solute partitioning, $k_{v}$, and the slope, $m_{v}$, of liquidus line in the kinetic phase diagram. The behavior of theses functions is rather different for the cases of local equilibrium and local non-equilibrium solute diffusion $[17,19,21,22]$.

\subsection{The second asymptotic: a case of slow solidification}

To predict condition of stability for the slowly moving planar interface, we consider small solidification velocity. With this aim, one can neglect quadratic terms by velocity $V$ and use the expression $G_{S}-G_{L} \sim V$ in equation (14). Also, for the slow-rate solidification regime, one can assume that the velocity dependent functions $k_{v}$ and $m_{v}$ merely have their own equilibrium constants $k_{e}$ and $m_{e}$, respectively. Therefore, equation for extrema of the function $S\left(\omega^{2}\right)$ is described by

$$
\frac{\partial S\left(\omega^{2}\right)}{\partial \omega^{2}}=-\Gamma+\frac{m G_{C}}{2} \frac{1}{\sqrt{(V / 2 D)^{2}+\omega^{2}}} \frac{k_{e} V / D}{\left(\omega_{C}-\left(1-k_{e}\right) V / D\right)^{2}}=0,
$$

where the frequency $\omega_{C}$ is given by (see Eq. (8) with neglecting quadratic terms by velocity $V$ )

$$
\omega_{C}=\frac{V}{2 D}+\sqrt{\left(\frac{V}{2 D}\right)^{2}+\omega^{2}}
$$


Using the new variable $r$ defined by

$$
r^{2}=\left(1+(2 D \omega / V)^{2}\right)^{1 / 2},
$$

equation (20) takes the following form

$$
-\Gamma+\frac{m G_{C}}{2} \cdot \frac{1}{(V / 2 D) r^{2}} \cdot \frac{k_{e} V / D}{\left((V / 2 D)\left(r^{2}+2 k_{e}-1\right)\right)^{2}}=0 .
$$

This equation can be easily re-written as follows

$$
r^{3}+\left(2 k_{e}-1\right) r-\left(2 k_{e} / A^{1 / 2}\right)=0,
$$

where the parameter $A$ is given by

$$
A=\frac{k_{e} \Gamma V^{2}}{m_{e} G_{C} D^{2}}
$$

Cubic polynomial (24) has been analyzed in details by Sekerka and Coriell in references [9,10]. In particular, defining $\mathcal{G}=\bar{G} /\left(m_{e} G_{C}\right)$, where $\bar{G}=\left(G_{L}+G_{S}\right) / 2$ is the weighted temperature gradient, and using equation (10), the following relationship at the onset of instability is found [9]:

$$
\mathcal{G}=1-\frac{3}{2} r A^{1 / 2}+\frac{A}{4 k_{e}}\left[1-\left(1-2 k_{e}\right) r^{2}\right]
$$

where $r$ is the one real root greater than unity of equation (24). For slow solidification, the parameter $A$ given by equation (25) is small compared to unity. Consequently, equation (26) leads to the following criterion [11]:

$$
\mathcal{G}=1
$$

which is known as the modified constitutional supercooling criterion [12] equals to

$$
\bar{G}=m_{e} G_{C} \equiv m C_{\infty} \frac{k_{e}-1}{k_{e}} \frac{V}{D},
$$

where equation (17) has been taken into account for small velocity, $V<<V_{D}$.

Equation (28) shows that the liquid ahead of the interface is constitutionally supercooled if $\bar{G}<m_{e} G_{C}$ that is leading to morphological instability of the planar solidification front. Neglecting the thermal gradient in solid and using $\bar{G}=G_{L}$, one can get from equation (28) the constitutional supercooling criterion in its classical form given by Ivantsov and Tiller et al. [23,24].

\section{Model with the deviations from local equilibrium at the interface and in solute diffusion field}

For computation of the morphological stability diagram we assume that characteristic size of pattern upon instability of the planar solidification interface is defined by the marginal stability hypothesis given by the analysis of Langer and Müller-Krumbhaar [25]. Following this hypothesis, the size $R$ of pattern is equal to the critical wavelength 
$\lambda$ of equation (1). Therefore, using, criterion (2), for a given velocity $V<V_{D}$ and temperature gradients $G_{L}$ and $G_{S}$, the pattern size $R$ is defined by

$$
R=\left(\frac{\Gamma / \sigma}{m_{v} G_{C} \xi_{c}-\frac{1}{2}\left(G_{L} \xi_{L}+G_{S} \xi_{S}\right)}\right)^{1 / 2}
$$

where $\sigma=\left(4 \pi^{2}\right)^{-1}$ is a parameter of marginal stability giving relation between $R$ and a wavelength of interfacial perturbation.

For the case of local non-equilibrium solute diffusion, the gradient $G_{C}$ is defined as follows [26]

$$
\begin{aligned}
& G_{C}=-\frac{V}{D} \frac{\left(1-k_{v}\right) C_{\infty}}{\left(1-V^{2} / V_{D}^{2}\right)\left(1-\left(1-k_{v}\right) \operatorname{Iv}\left(P_{C}\right)\right)}, \quad V>V_{D}, \\
& G_{C}=0, \quad V \geq V_{D},
\end{aligned}
$$

where $P_{C}=V R / 2 D$ is the solutal Peclet number. The $\operatorname{Ivantsov-function} \operatorname{Iv}(P)$ is defined by:

$$
\begin{aligned}
& \operatorname{Iv}(P)=(\pi P)^{1 / 2} \exp (P) \operatorname{erfc}\left(P^{1 / 2}\right), \quad \text { for } 2 \mathrm{D}, \\
& \operatorname{Iv}(P)=P \exp (P) \mathrm{E}_{\mathrm{i}}(P), \quad \text { for } 3 \mathrm{D},
\end{aligned}
$$

where $\mathrm{E}_{\mathrm{i}}(P)=\int_{P}^{\infty} s^{-1} \exp (-s) d s$ is the exponential integral function. The stability functions $\xi$ can be obtained from equations (3)-(5) as follows [17]

$$
\begin{aligned}
& \xi_{L}=1-\frac{1}{\left(1+\frac{1}{\sigma P_{T}^{2}}\right)^{1 / 2}}, \\
& \xi_{S}=1+\frac{1}{\left(1+\frac{1}{\sigma P_{T}^{2}}\right)^{1 / 2}}, \\
& \xi_{C}=1+\frac{2 k_{v}}{1-2 k_{v}-\left(1+\frac{1-V^{2} / V_{D}^{2}}{\sigma P_{C}^{2}}\right)^{1 / 2}}, V<V_{D}, \\
& \xi_{C}=0, \quad V \geq V_{D},
\end{aligned}
$$

where $P_{T}=V R / 2 a$ is the thermal Peclet number.

The slope $m_{v}$ of the nonequilibrium liquidus is described by [21]:

$$
\begin{aligned}
& m_{v}=\frac{m_{e}}{1-k_{e}}\left\{1-k_{v}+\ln \left(\frac{k_{v}}{k_{e}}\right)+\left(1-k_{v}\right)^{2} \frac{V}{V_{D}}\right\}, V<V_{D}, \\
& m_{v}=\frac{m_{e} \ln k_{e}}{k_{e}-1}, \quad V \geq V_{D} .
\end{aligned}
$$

The solute distribution function $k_{v}$ at the interface defines the solute trapping and it is described by [27]:

$$
k_{v}=\frac{\left(1-V^{2} / V_{D}^{2}\right)\left[k_{e}+\left(1-k_{e}\right) C_{\infty}\right]+V / V_{D I}}{1-V^{2} / V_{D}^{2}+V / V_{D I}}, V<V_{D},
$$


Table 1. Physical parameters of $\mathrm{Si}-\mathrm{Sn}$ alloy used in calculations of the morphological stability diagrams.

\begin{tabular}{lcccc}
\hline Parameter & Notation & Dimension & Values & Reference \\
\hline Thermal conductivity & $K_{L}$ & $\mathrm{~W} / \mathrm{K} / \mathrm{m}$ & 140 & {$[30]$} \\
Heat capacity & $c_{p}$ & $\mathrm{~J} / \mathrm{K} / \mathrm{m}^{3}$ & $2.5 \times 10^{6}$ & {$[30]$} \\
Diffusion coefficient & $D$ & $\mathrm{~m}^{2} / \mathrm{s}$ & $2.5 \times 10^{-8}$ & {$[31]$} \\
Partition coefficient & $k_{e}$ & - & 0.016 & {$[31]$} \\
Liquidus slope & $m_{e}$ & $\mathrm{~K} / \mathrm{at} . \%$ & -4.6 & {$[31]$} \\
Gibbs-Thomson coefficient & $\Gamma$ & $\mathrm{Km}$ & $1.3 \times 10^{-7}$ & {$[31]$} \\
Interface diffusion speed & $V_{D I}$ & $\mathrm{~m} / \mathrm{s}$ & 17 & {$[31]$} \\
Diffusion speed in bulk liquid & $V_{D}$ & $\mathrm{~m} / \mathrm{s}$ & 17.5 & {$[17]$} \\
\hline
\end{tabular}

$$
k_{v}=1, \quad V \geq V_{D}
$$

where $V_{D I}$ is the interfacial diffusion speed $[28,29]$ which is usually assumed to be $V_{D I}<V_{D}$.

As a result, equations (29)-(36) generalize the model of Kurz, Giovanola, and Trivedi (KGT-model) [15] for the case of deviation from local equilibrium in bulk liquid (i.e., in the solute diffusion field). The KGT-model itself directly follows from equations (30)-(36) in the limits $V_{D} \rightarrow \infty$.

\section{Morphological stability diagram}

For the further analysis, we assume that $(i)$ the solidification proceeds in the positive temperature gradient in the liquid and one can neglect the gradient in the solid, so that $G_{L}>0$ and $G_{S}=0$ and $(i i)$ all calculations will be made for material parameters of $\mathrm{Si}-\mathrm{Sn}$ alloy summarized in Table 1.

\subsection{Critical values of parameters}

With a given velocity $V$ and for various gradients $G_{L}$, equation (29) may have the only solution, two solutions, or no any solutions. These three situations are shown in Figure 2 using the dashed-dotted line, dashed line, and dotted line, respectively. If $G_{L}$ is smaller than some critical value $G^{*}(V)$, then equation (29) has two roots the minima of which give the selected size of crystalline microstructure. As the temperature gradient increases up to the critical value $G^{*}(V)$, equation (29) has the only root which gives the maximal characteristic size of the microstructure at a given interface velocity $V$. Further increasing the gradient $G_{L}$ leads to the absence of any solution for equation (29) that gives a stability of the planar interface with $R \rightarrow \infty$. Thus, a curve for the critical temperature gradient $G^{*}(V)$ defines $V$ and $G_{L}$ at which the transition from planar front to cells and dendrites or vice versa may occur in a binary alloy.

\subsection{The complete diagram of morphology}

Figure 3 presents morphological stability diagram which is known as "nose-like diagram of interfacial stability" [32,33]. The diagram shows regions of planar front stability and of cells/dendrites existence for given nominal concentration and interface 


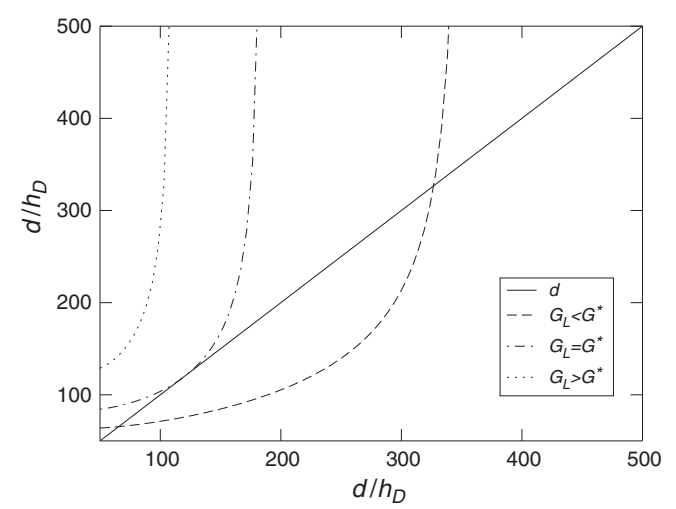

Fig. 2. Dependence of left hand side (solid line) and right hand side (dotted, dashed and dashed-dotted) of equation (29) on characteristic size $R$ of microstructure. The curves are plotted for given velocity $V$ and various gradients $G_{L}$. The size $R$ is scaled by the diffusion length $h_{D}=D / V_{D}$.

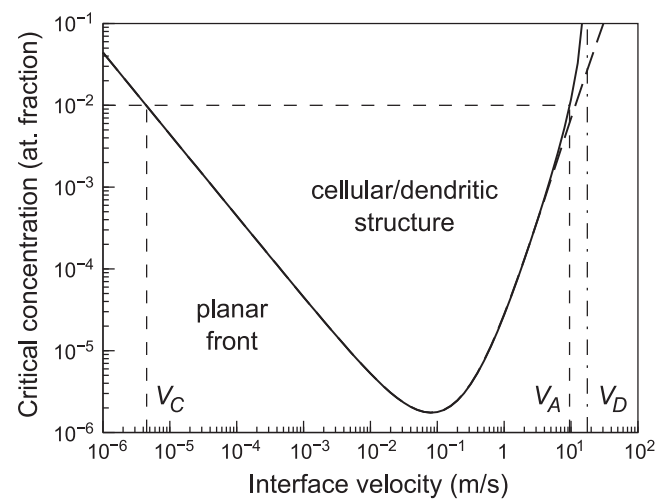

Fig. 3. The complete morphological stability diagram in a form of critical concentration, $C_{\infty}(V)$, above which planar interface is unstable (solid line) for a given temperature gradient $G_{L}=10^{5} \mathrm{~K} / \mathrm{m}$. The limiting velocity $V_{C}$ is given by equation (28) and the limiting velocity $V_{A}$ is given by equations (19), (35) and (36). Dashed-dotted line, $V=V_{D}$, represents the limiting velocity for the absolute interface stability.

velocity $V$. The most important issue that the diagram predicts critical value for the transitions between growing structures in steady state crystalline solidification obtained by the constitutional criterion (28) at small $V$ and absolute chemical stability condition (19), (35) and (36) given by the high $V$-limit. In addition to the structure diagram calculated in reference [17] the diagram in Figure 3 represents the whole range of the front velocities from its low to high values at which morphological transitions may occur at the corresponding values of the critical concentration.

\subsection{High velocity limit and dependence on temperature gradient}

The high $V$-limit given in the complete diagram principally differs from the previously obtained in references [32]. Indeed, as Figure 4 shows (which is zoom of Fig. 3 for the region of high growth velocity), the model with the deviation from local equilibrium only at the interface predicts gradual increasing of concentration with no sensetive to the bulk diffusion speed (dashed line). By contrast, the model with deviation 


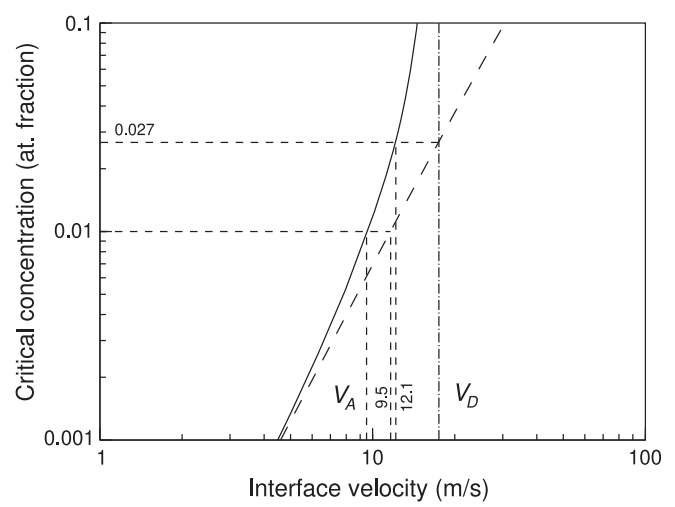

Fig. 4. A part of the morphological stability diagram for high solidification rates given by equations (19), (35) and (36).

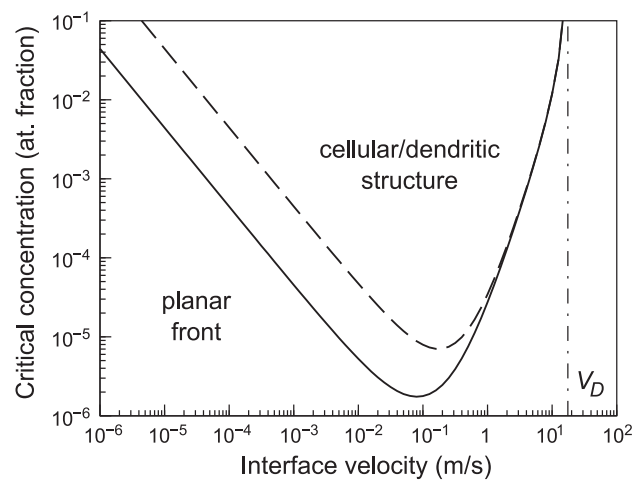

Fig. 5. Dependence of the morphological stability from the temperature gradient as predicted by equations (19), (35) and (36). The curves are computed for various temperature gradients: solid line is given by $G_{L}=10^{5} \mathrm{~K} / \mathrm{m}$, and dashed line is given by $G_{L}=10^{6} \mathrm{~K} / \mathrm{m}$. Dashed-dotted line, $V=V_{D}$, represents the limiting velocity for the absolute interface stability.

from local equilibrium at the interface and in bulk diffusion field is limited by the diffusion speed in bulk liquid and one gets $V_{A}<V_{D}$. As a result the curve of critical concentration (solid line in Fig. 4) essentially deviates from that one given by the model with the deviation from local equilibrium only at the interface (i.e. from the dashed line in Fig. 4). Such deviation around $V=V_{A}$ gradually increases and leads to essential difference inpredictions of the growth kinetics of dendrites [18]. One can note that for the diffusion coefficient $D$, equilibrium partition coefficient $k_{e}$ and the liquidus line slope $m_{e}$ we have used in calculations their constant values independent on temperature and concentration (see Tab. 1). In a wide range of undercooling and for a dilute and concentrated alloys these material parameters should be taken as functions of temperature and concentration that is, of course, may correct the morphological diagram shown in Figures 3 and 5.

Finally, one has to note that the temperature gradient $G_{L}$ mainly influences the critical concentration for the morphology transition at small growth velocity. Figure 5 shows that the increase in $G_{L}>0$ leads to the increase in the region for the planar front stability at small velocity $V$. This fact follows from the criterion of constitutional 
undercooling (28) if it can be rewritten in the form

$$
C_{\infty}=\frac{k_{e}}{m_{e}\left(k_{e}-1\right)} \cdot \frac{D G_{L}}{2 V}, \text { where } \overline{\mathrm{G}}=\mathrm{G}_{\mathrm{L}} / 2
$$

which predicts that the nominal concentration $C_{\infty}$ should be increased as the temperature gradient $G_{L}$ increases for a given interface velocity $V$. By contrast, the high velocity limit does not strongly depend on the gradient $G_{L}$. It appears due to definition of absolute chemical stability: the criterion (19) is the result of competition and balance of the capillary length and solute diffusion length that does not depend of the imposed temperature gradient directly.

\section{Conclusions}

Using the criterion of marginal stability we found the conditions of constitutional undercooling, which acts at small solidification rates, and the absolute chemical stability as a result of the competition between contributions of surface energy and destabilizing concentration gradient. These conditions are related to general solidification regimes existing at very small and very high solid-liquid interface velocity, which occur under deviation from local equilibrium at the interface and in the diffusion profile in bulk liquid. As well as these conditions present in asymptotic cases of the complete regimes obtained from the diagram of morphologies computed using the formulated model of dendritic/cellular growth.

The constructed morphology diagram predicts critical values of concentration and interface velocity dividing the region of planar solidification front stability and the region of cellular/dendritic structures growing in a positive temperature gradient during slow, intermediate and rapid directional solidification of binary alloys. Combinations of the full diagram of structures and dynamic conditions of the existence of stationary or non-stationary modes of solidification complement the interpretation of experimental results [34].

Open access funding provided by Projekt DEAL. The authors acknowledge the support by the European Space Agency (ESA) under research project MULTIPHAS (AO-2004), the German Aerospace Center (DLR) Space Management under contract No. 50WM1541 and also from the Russian Science Foundation under the project no. 16-11-10095.

\section{Author contribution statement}

All authors contributed equally to the present research article.

Open Access This is an open access article distributed under the terms of the Creative Commons Attribution License (http://creativecommons.org/licenses/by/4.0), which permits unrestricted use, distribution, and reproduction in any medium, provided the original work is properly cited.

\section{References}

1. V.T. Borisov, Theory of two-phase mushy zone of metallic ingot (Metallurgia, Moscow, 1987) 
2. V.A. Zhuravlev, Solidification and crystallization of alloys with hetero-trasformations (Regular and Chaotic Dynamics, Izhevsk, 2006)

3. V.T. Borisov, Yu.E. Matveev, Fizika Metallov i Metallovedenie 13, 465 (1962)

4. R. Trivedi, W. Kurz, Int. Mater. Rev. 39, 49 (1994)

5. W. Kurz, D.J. Fisher, R. Trivedi, Int. Mater. Rev. 64, 311 (2019)

6. P.K. Galenko, D.V. Alexandrov, Philos. Trans. R. Soc. A 376, 20170210 (2018)

7. W.W. Mullins, R.F. Sekerka, J. Appl. Phys. 34, 323 (1963)

8. W.W. Mullins, R.F. Sekerka, J. Appl. Phys. 35, 444 (1964)

9. R.F. Sekerka, J. Appl. Phys. 36, 264 (1965)

10. S.R. Coriell, R.F. Sekerka, in Rapid solidification processing II, edited by R. Mehrabian, B.H. Kear, M. Cohen (Claitor's, Baton Rouge, Louisiana, 1980), p. 35

11. S.R. Coriell, G.B. McFadden, R.F. Sekerka, Annu. Rev. Mater. Sci. 15, 119 (1985)

12. S.R. Coriell, G.B. McFadden, in Handbook of crystal growth, edited by D.T.J. Hurl (Elsevier, Amsterdam, 1993), Vol. 1a, p. 785

13. S.H. Davis, Theory of solidification (Cambridge University Press, Cambridge, UK, 2001)

14. R. Trivedi, W. Kurz, Acta Metall. 34, 1663 (1986)

15. W. Kurz, B. Giovanola, R. Trivedi, Acta Metall. 34, 823 (1986)

16. W. Kurz. D.J. Fisher, Fundamentals of solidification, 3rd edn. (Trans Tech Publications, Aedermannsdorf, 1992)

17. P.K. Galenko, D.A. Danilov, Phys. Rev. E 69, 051608 (2004)

18. D.M. Herlach, P.K. Galenko, D. Holland-Moritz, Metastable solids from undercooled melts, (Elsevier, Amsterdam, 2007)

19. P.K. Galenko, D.A. Danilov, J. Cryst. Growth 216, 512 (2000)

20. W.J. Boettinger, S.R. Coriell, R.F. Sekerka, Mater. Sci. Eng. A 65, 27 (1984)

21. P. Galenko, Phys. Rev. B 65, 144103 (2002)

22. P.K. Galenko, Mater. Sci. Eng. A 375-377, 493 (2004)

23. G.P. Ivantsov, Dokl. Akad. Nauk SSSR 81, 179 (1951)

24. W.A. Tiller, K.A. Jackson, J.W. Rutter, B. Chalmers, Acta Metall. 428, 1 (1953)

25. J.S. Langer, H. Müller-Krumbhaar, Acta Metall. 26, 1681 (1978)

26. P.K. Galenko, D.A. Danilov, Phys. Lett. A 235, 271 (1997)

27. P. Galenko, Phys. Rev. E 76, 031606 (2007)

28. M.J. Aziz, T. Kaplan, Acta Metall. 36, 2335 (1988)

29. M.J. Aziz, J. Appl. Phys. 53, 1158 (1982)

30. D.E. Hoglund, M.O. Thompson, M.J. Aziz, Phys. Rev. B 58, 189 (1998)

31. D.E. Hoglund, M.J. Aziz, Mater. Res. Soc. Symp. Proc. 205, 325 (1992)

32. D.E. Hoglund, M.J. Aziz, S.R. Stiffler, M.O. Thompson, J.Y. Tsao, P.S. Peercy, J. Cryst. Growth 109, 107 (1991)

33. H. Biloni, W.J. Boettinger, Solidification, in Physical metallurgy, edited by R.W. Cahn, P. Haasen, (Elsevier Science Publishers, Amsterdam, 1996), Vol. I, Chap. 8, pp. 669-842

34. K. Reuther, P.K. Galenko, M. Rettenmayr, J. Cryst. Growth 506, 55 (2019) 